OPEN ACCESS

Edited by:

Liang Cheng,

Harbin Medical University, China

Reviewed by:

Kristen M. Tecson

Baylor Scott \& White Research Institute (BSWRI), United States

Xinghao $\mathrm{Yu}$,

Soochow University, China

*Correspondence:

Shilong Zhong

gdph_zhongs/@gd.gov.cn

†These authors have contributed equally to this work and share first

authorship

Specialty section:

This article was submitted to Heart Failure and Transplantation,

a section of the journal

Frontiers in Cardiovascular Medicine

Received: 15 April 2021

Accepted: 10 August 2021

Published: 14 September 2021

Citation:

Wang Z, Chen S, Zhu Q, Wu Y, Xu G,

Guo G, Lai W, Chen J and Zhong $S$ (2021) Using a Two-Sample Mendelian Randomization Method in Assessing the Causal Relationships Between Human Blood Metabolites and Heart Failure.

Front. Cardiovasc. Med. 8:695480. doi: 10.3389/fcvm.2021.695480

\section{Using a Two-Sample Mendelian Randomization Method in Assessing the Causal Relationships Between Human Blood Metabolites and Heart Failure}

\author{
Zixian Wang ${ }^{1,2,3 \dagger}$, Shiyu Chen ${ }^{1,2,37}$, Qian Zhu ${ }^{2,3,4}$, Yonglin $W u^{2,3,5}$, Guifeng $X u^{1,2}$, \\ Gongjie Guo ${ }^{2,3,4}$, Weihua Lai ${ }^{2}$, Jiyan Chen ${ }^{3}$ and Shilong Zhong ${ }^{1,2,3,4,5 *}$ \\ ${ }^{1}$ School of Biology and Biological Engineering, South China University of Technology, Guangzhou, China, ${ }^{2}$ Department of \\ Pharmacy, Guangdong Provincial People's Hospital, Guangdong Academy of Medical Sciences, Guangzhou, China, \\ ${ }^{3}$ Guangdong Provincial Key Laboratory of Coronary Heart Disease Prevention, Guangdong Cardiovascular Institute, \\ Guangdong Provincial People's Hospital, Guangdong Academy of Medical Sciences, Guangzhou, China, ${ }^{4}$ Guangdong \\ Provincial People's Hospital, Guangdong Academy of Medical Sciences, School of Medicine, South China University of \\ Technology, Guangzhou, China, ${ }^{5}$ School of Pharmaceutical Sciences, Southern Medical University, Guangzhou, China
}

Background: Heart failure (HF) is the main cause of morbidity and mortality worldwide, and metabolic dysfunction is an important factor related to HF pathogenesis and development. However, the causal effect of blood metabolites on HF remains unclear.

Objectives: Our chief aim is to investigate the causal relationships between human blood metabolites and HF risk.

Methods: We used an unbiased two-sample Mendelian randomization (MR) approach to assess the causal relationships between 486 human blood metabolites and HF risk. Exposure information was obtained from Sample 1, which is the largest metabolome-based genome-wide association study (mGWAS) data containing 7,824 Europeans. Outcome information was obtained from Sample 2, which is based on the results of a large-scale GWAS meta-analysis of HF and contains 47,309 cases and 930,014 controls of Europeans. The inverse variance weighted (IVW) model was used as the primary two-sample MR analysis method and followed the sensitivity analyses, including heterogeneity test, horizontal pleiotropy test, and leave-one-out analysis.

Results: We observed that 11 known metabolites were potentially related to the risk of HF after using the IVW method $(P<0.05)$. After adding another four MR models and performing sensitivity analyses, we found a 1-SD increase in the xenobiotics 4-vinylphenol sulfate was associated with $\sim 22 \%$ higher risk of HF (OR [95\%Cl], 1.22 [1.07-1.38]).

Conclusions: We revealed that the 4-vinylphenol sulfate may nominally increase the risk of HF by $22 \%$ after using a two-sample MR approach. Our findings may provide novel insights into the pathogenesis underlying HF and novel strategies for HF prevention.

Keywords: heart failure, blood metabolites, Mendelian randomization, 4-vinylphenol sulfate, causal relationship 


\section{INTRODUCTION}

Heart failure (HF) is a major public health problem and has imposed considerable burden on society (1). Although great progress has been made in current treatment of HF, its morbidity and mortality continue to rise (2). HF is estimated with a heritability of $\sim 26 \%$ (3). Previous genomewide association studies (GWAS) have identified a few genetic loci for HF (4), while its roles in etiology are unclear. As functional intermediates, circulating metabolites can reflect the underlying biological links of the individual genetic composition and the development of diseases. To date, metabolic dysfunction was proposed as an important contributor in HF (5), and metabolomic studies have identified a number of circulating metabolites associated with $\operatorname{HF}(6,7)$. However, the causal relationships between the metabolites and HF are unclear, and translating these metabolic findings into pathophysiological mechanisms and novel therapies is difficult. Hence, a comprehensive analysis is needed to uncover the interactions between genetics and circulating metabolites in the pathogenesis of HF.

The basic idea of Mendelian randomization (MR) is to use genetic variation as an instrumental variable (IV), which is strongly related to exposure factors and can infer the causal effects between exposure factors and research outcomes (8). To date, some MR studies have been performed in exploring the causation between exposure and heart failure, though the main focus was single exposure or routine exposure factors, such as brain natriuretic peptide (9), interleukin-6 (10), and heart rate (11). Few studies focused on the blood metabolites, especially based on the metabolome. A previous study conducted two-sample MR analysis on 486 blood metabolites and five major psychiatric disorders. It has successfully identified several disease-linked metabolites (12), providing novel insights into integrating metabolic mechanism with psychiatric disorders. However, no research about investigating the causal relationships between blood metabolites and the risk of HF has been reported. Hence, we used a two-sample MR approach for assessing the causal relationships between 486 human blood metabolites and risk of HF in this study to provide a deeper understanding of the pathogenesis of HF.

\section{METHODS}

\section{Study Design and Data Resources}

The data we used in this study all came from the public dataset, which are publicly available on the database website, and has obtained ethics approval in the previous studies.

The study flow is illustrated in Figure 1. Exposure information was obtained from Sample 1, which is the largest mGWAS data published by Shin et al. (13) in 2014 and contains 7,824 Europeans. After strict quality control, $\sim 2.1$ million

Abbreviations: HF, heart failure; MR, Mendelian randomization; mGWAS, metabolome-based genome-wide association study; GWAS, genome-wide association study; IV, instrumental variable; SNP, single nucleotide polymorphism; LD, linkage disequilibrium; IVW, inverse variance weighted. single nucleotide polymorphisms (SNPs) and 486 blood metabolites (including 309 known metabolites and 177 unknown metabolites) were employed. These metabolites can be split into eight major categories: carbohydrates, amino acids, nucleotides, cofactors and vitamins, lipids, peptides, energy products, and xenobiotic metabolites. Summary data of all the mGWAS results in Sample 1 are publicly available on a database website (http:// metabolomics.helmholtz-muenchen.de/gwas/).

Outcome information was obtained from Sample 2, which is based on the results of a large-scale GWAS meta-analysis conducted by Shah et al. (4) in 2020 on 26 studies of HF. This dataset contains 47,309 cases and 930,014 controls of European lineage, and $\sim 8.3$ million SNPs were employed in association analyses. The summary data of HF GWAS in Sample 2 were downloaded from the CVDKP Datasets website (http://www. $\mathrm{kp} 4 \mathrm{~cd}$.org/datasets/mi).

\section{Quality Control of IV}

A series of unified selection standards was adopted for the genetic variation in 486 metabolites in this study. We used a relatively loose $P$-value threshold, which was widely used in MR analysis (8), that is, $P<1 \times 10^{-5}$, as a significant condition for the preliminary selection of IVs. Then, we performed linkage disequilibrium (LD) analysis to achieve independent genetic instruments, which were derived from a stringent clumping criterion [LD cutoff of $r^{2}=0.001$ within a $10,000 \mathrm{~kb}$ window in the 1000 Human Genomes Project (14) European (EUR) reference panel]. Given that metabolites in similar metabolic pathways may be regulated by the same SNPs and multiple metabolites are significantly associated with the same IVs, so the MR hypothesis could be disturbed. Hence, we conducted the restricted selection of IVs (15) to exclude SNPs that were significantly related to more than two metabolites. Besides, we searched for keywords [(HF) OR (heart failure) AND (SNP) OR (GWAS)] in the PubMed, and we collected SNPs related to HF (including various types, e.g., dilated cardiomyopathy, incident systolic heart failure, advanced heart failure, congestive hearts failure) or its risk factors (such as interleukin-6, ejection fraction, heart rate, aortic root size, etc.) in the published literature. We deleted the disease-related SNPs (Supplementary Table 1) and the duplicate SNPs after sorting and merging. Finally, we used the unique SNP for subsequent analysis.

\section{MR Analysis}

The inverse variance weighted (IVW) model was used as the primary two-sample MR analysis model. IVW was proposed by Burgess et al. (16) and usually used in the MR studies of multiple IVs. This method can be employed on the premises that IVs satisfy the assumptions of relevance, independence, and exclusivity and genetic variation affect outcomes only through exposure in the study. The IVW method is ideal in estimating robust causal detection ability. We considered that the features of these metabolites and HF risk have a strong causal relationship if the $P$-value of IVW exceeds the multiple-testing adjusted threshold $\left(P<0.05 / 486=1.03 \times 10^{-4}\right)$. However, given that the causal effects between blood metabolites and risk of HF sometimes are limited, a strict threshold might lead to the loss of 


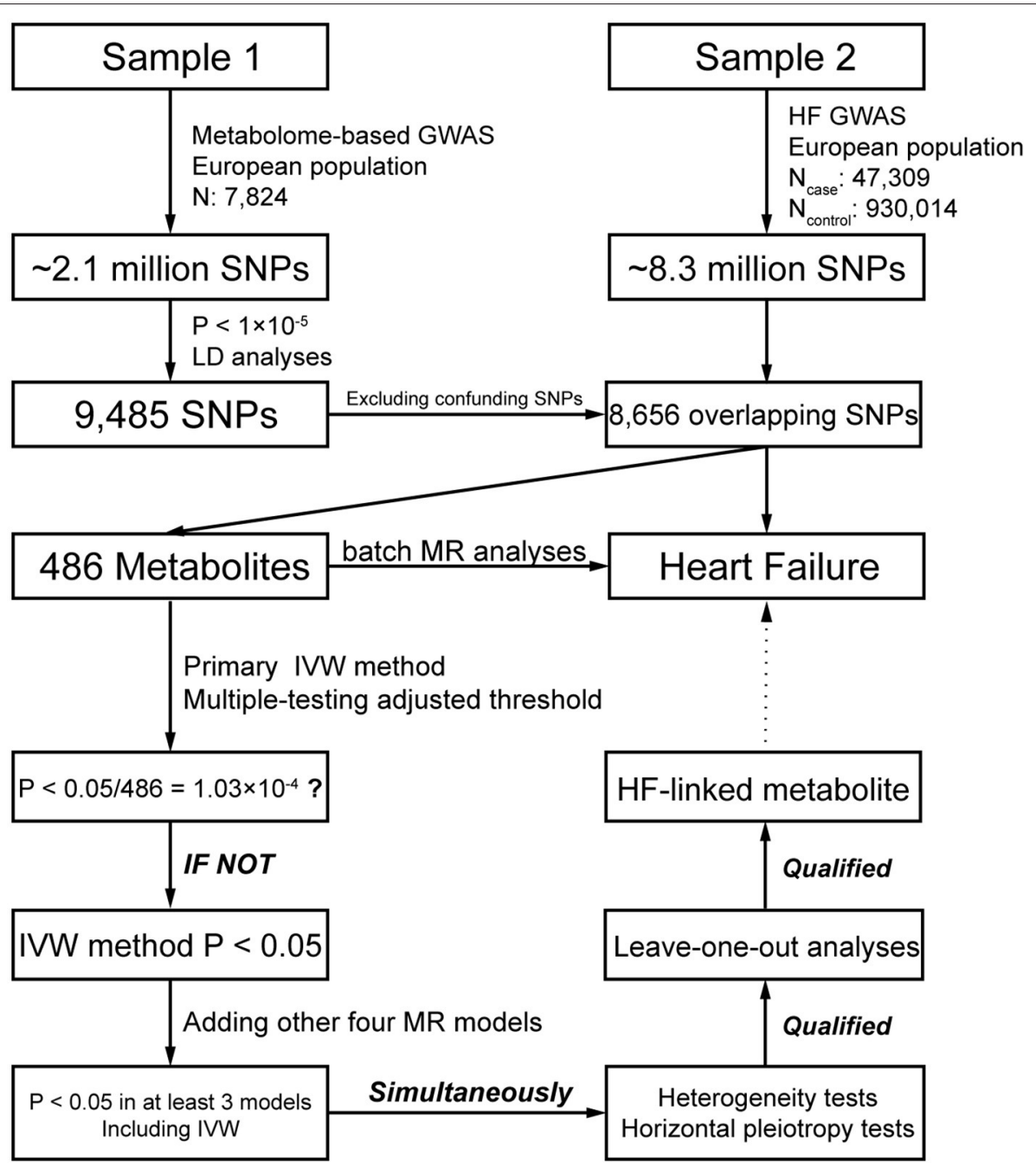

FIGURE 1 | Schematic view of the study design for two-sample MR analyses in this study.

some potential signals. Hence, we focused on potentially causal metabolites $\left(P>1.03 \times 10^{-4}\right.$ but $\left.P<0.05\right)$ and added four extra MR models to test the causal influence features, namely, MR-Egger regression (17), the weighted median method (18), the simple mode-based estimator (19), and the weighted mode-based estimator (19).

MR-Egger regression is the weighted linear regression of the effects of IVs and exposure and the effects of IVs and outcome (17). Different from the intercept term of IVW forced linear regression, the latter is zero, the intercept term in this model is a variable, and the horizontal pleiotropy of genetic variation can be measured by the intercept term. The fact that the intercept term does not correspond to zero indicates pleiotropy, but MR-Egger can still get an unbiased estimation when the IVs exist pleiotropy, which is its advantage. When applying the MR-Egger model, the tool variables will have nothing to do with the outcome, and only through exposure factors affect the outcome, which weakens the exclusive hypothesis of the IVM method to the tool variables. MR-Egger just needs to meet the hypothesis named "InSIDE (instrument strength independent of direct effect) assumption" that the precise effects of tool variables and outcomes are independent of the correlation between tool variables and exposure factors. It is to be noted that the direction of all tool variables is the same in the analysis. Although the assumption of IVs can be effectively evaluated through the intercept item of MR Egger, it is less effective than IVW approach in detecting the causality (20).

The weighted median method is generally employed in measuring an effect, and the ratios of selected SNPs are calculated for the estimation of a weighted empirical distribution function (18). This method allows a strong SNP to provide an asymptotically consistent estimate of causal effects; even when an effective SNP is less, it can also reduce the bias of causal effects estimation.

The simple mode-based estimator classifies SNPs according to causal effects, and similar values are divided into a cluster. The 
TABLE 1 | Significant metabolites related to the risk of HF according to IWW results $(P<0.05)$.

\begin{tabular}{|c|c|c|c|c|c|c|}
\hline ID & Metabolite & nSNP & Beta & SE & $\boldsymbol{P}$ & OR $(95 \% \mathrm{Cl})$ \\
\hline M36098 & 4-vinylphenol sulfate & 10 & 0.20 & 0.06 & 2.16E-03 & $1.22(1.07-1.38)$ \\
\hline M35187 & $X-13429$ & 4 & 0.27 & 0.09 & 3.10E-03 & $1.32(1.10-1.58)$ \\
\hline M34035 & Linolenate [alpha or gamma; (18:3n3 or 6)] & 3 & 0.54 & 0.20 & 6.31E-03 & $1.71(1.16-2.52)$ \\
\hline M34336 & $X-12726$ & 22 & 0.12 & 0.05 & 7.15E-03 & $1.13(1.03-1.23)$ \\
\hline M35160 & Oleoylcarnitine & 7 & 0.47 & 0.18 & 8.06E-03 & $1.60(1.13-2.28)$ \\
\hline M34112 & $X-12544$ & 18 & -0.15 & 0.06 & 1.06E-02 & $0.86(0.77-0.97)$ \\
\hline M01125 & Isoleucine & 16 & 0.73 & 0.29 & $1.11 \mathrm{E}-02$ & $2.08(1.18-3.67)$ \\
\hline M16818 & X-04495 & 11 & 0.35 & 0.14 & $1.32 \mathrm{E}-02$ & $1.42(1.08-1.87)$ \\
\hline M35159 & Cysteine-glutathione disulfide & 9 & -0.19 & 0.08 & 1.45E-02 & $0.82(0.71-0.96)$ \\
\hline M18477 & Glycodeoxycholate & 7 & -0.11 & 0.05 & $1.53 \mathrm{E}-02$ & $0.90(0.82-0.98)$ \\
\hline M33138 & $X-11793$ & 11 & 0.30 & 0.13 & 1.67E-02 & $1.35(1.06-1.73)$ \\
\hline M33782 & $X-10346$ & 14 & 0.08 & 0.04 & $1.81 \mathrm{E}-02$ & $1.09(1.01-1.17)$ \\
\hline M12768 & X-03088 & 16 & -0.30 & 0.13 & 2.43E-02 & $0.74(0.57-0.96)$ \\
\hline M33203 & $X-11858$ & 15 & 0.06 & 0.03 & 2.92E-02 & $1.06(1.01-1.12)$ \\
\hline M35186 & 1-arachidonoylglycerophosphoethanolamine & 17 & -0.32 & 0.15 & 3.05E-02 & $0.72(0.54-0.97)$ \\
\hline M33192 & $X-11847$ & 11 & 0.10 & 0.05 & 3.31E-02 & $1.11(1.01-1.22)$ \\
\hline M11438 & Phosphate & 4 & -0.84 & 0.40 & $3.40 \mathrm{E}-02$ & $0.43(0.20-0.94)$ \\
\hline M35464 & $X-13671$ & 14 & -0.38 & 0.18 & 3.45E-02 & $0.68(0.48-0.97)$ \\
\hline M34530 & $X-12847$ & 10 & 0.13 & 0.06 & $4.22 \mathrm{E}-02$ & $1.14(1.00-1.30)$ \\
\hline M33973 & Epiandrosterone sulfate & 3 & 0.20 & 0.10 & 4.31E-02 & $1.22(1.01-1.49)$ \\
\hline M01114 & Deoxycholate & 17 & 0.11 & 0.05 & 4.41E-02 & $1.11(1.00-1.24)$ \\
\hline M33968 & 5-Dodecenoate (12:1n7) & 11 & -0.18 & 0.09 & 4.95E-02 & $0.83(0.70-1.00)$ \\
\hline
\end{tabular}

nSNP, number of the SNP used for tests; SE, standard error; OR, odds ratio; 95\% Cl, 95\% confidence interval; X, unknown metabolite.

estimated causal effect is estimated by a cluster with the largest number of SNPs. The weighted mode-based estimator weighs the causal effects values of each SNP pair to the number of SNPs in each cluster, and the results returned are temporary estimates with the maximum number of SNPs weight. The premise of using Mode-based Estimate method to access the consistent estimation of causal effect is to satisfy the "ZEMPA hypothesis" (Zero Modal Pleiotropy Assumption), that is, in the total genetic variation, the mode of the bias term is 0 (19).

In brief, if the five MR models mentioned above produce similar estimates of causal effects and show significant $P$-values $(P<0.05)$ in at least three models (including IVW), then we consider the metabolite as a candidate causal feature for HF risk.

\section{Sensitivity Analysis}

Owing to the diversity of experimental conditions, analytical platforms, and study subjects, there may be heterogeneity in the two-sample MR analyses, resulting in bias in the estimation of causal effects. Thus, heterogeneity testing of IVW analysis and MR-Egger regression was adopted in this study. If the $P>0.05$ in the test, evidence of heterogeneity in the included IVs is nonexistent, that is, the influence of heterogeneity on the estimation of causal effects can be ignored.

When we use IVW to explore the causal relationship, there may be other unknown confounding factors against genetic multiplicity and bias estimation of causal effects. Hence, we performed horizontal pleiotropy test by judging the intercept of MR-Egger regression and evaluating the $P$-value of it on the
MR-Egger model. If the intercept is close to $0(<0.1)$ and $P>$ 0.05 , we considered that there is no evidence for the existence of horizontal pleiotropy in the tests. In addition, we adopted MRPRESSO method to further test horizonal pleiotropy and possible outliers by using MR-PRESSO package (21).

After implementing the heterogeneity test and horizontal pleiotropy test, we used the leave-one-out method in conducting sensitivity analysis on qualified metabolites. In this method, related SNPs are removed one by one, and the amalgamation effect of the remaining SNPs is calculated for the evaluation of the effect of each SNP on the metabolites. If the overall error line does not change considerably after the exclusion of each SNP (i.e., all error lines do not pass through 0), the result is considered reliable.

\section{Pathway and Enrichment Analysis}

We performed pathway and enrichment analysis of $11 \mathrm{HF}-$ related known metabolites ( $P<0.05$, IVW method) through the online metabolomics data analysis website [(22); https://www. metaboanalyst.ca/MetaboAnalyst/faces/home.xhtml]. First, we found the ID corresponding to these metabolites on the Human Metabolome Database [(23); https://hmdb.ca/]. Then, we used Enrichment Analysis and Pathway Analysis modules in the Annotated Features mode to perform the pathway and enrichment analysis. Overall, we collected a number of metabolite sets and pathways of metabolites related to $\mathrm{HF}$ based on SMPDB [(24); https://smpdb.ca/] and KEGG database [(25); https://www.kegg.jp/]. 
TABLE 2 | Five MR models estimate the causal relationships between 11 known metabolites and the risk of HF and tests for heterogeneity and horizontal pleiotropy.

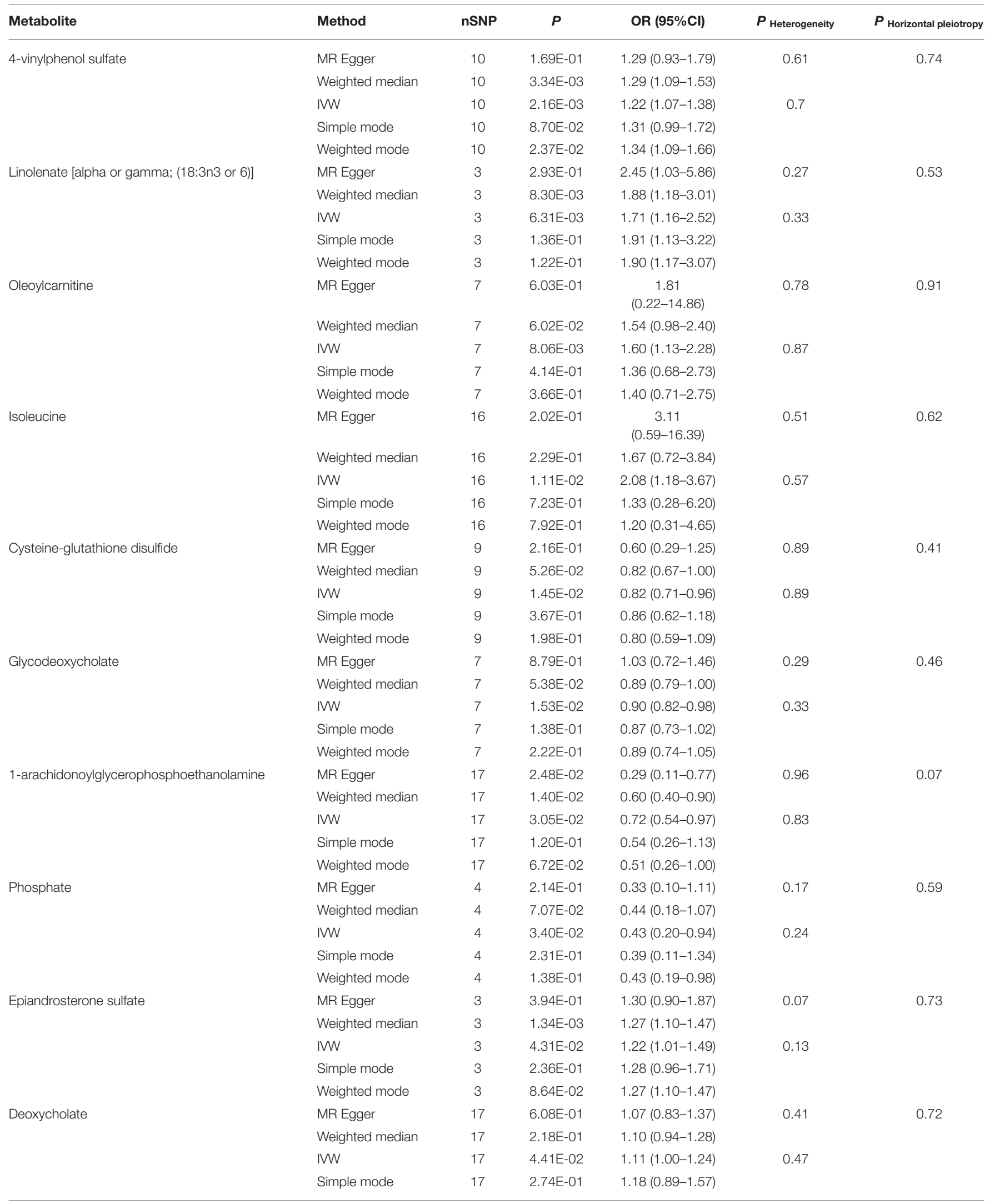


TABLE 2 | Continued

\begin{tabular}{|c|c|c|c|c|c|c|}
\hline Metabolite & Method & nSNP & $P$ & OR $(95 \% \mathrm{Cl})$ & $\boldsymbol{P}_{\text {Heterogeneity }}$ & $\boldsymbol{P}_{\text {Horizontal pleiotropy }}$ \\
\hline & Weighted mode & 17 & $3.11 \mathrm{E}-01$ & $1.15(0.89-1.49)$ & & \\
\hline \multirow[t]{5}{*}{ 5-dodecenoate (12:1n7) } & MR Egger & 11 & $2.28 \mathrm{E}-01$ & $0.78(0.54-1.14)$ & 0.75 & 0.7 \\
\hline & Weighted median & 11 & 3.68E-02 & $0.78(0.61-0.98)$ & & \\
\hline & IVW & 11 & 4.95E-02 & $0.83(0.70-1.00)$ & 0.81 & \\
\hline & Simple mode & 11 & 2.60E-01 & $0.79(0.53-1.17)$ & & \\
\hline & Weighted mode & 11 & 1.48E-01 & $0.79(0.60-1.06)$ & & \\
\hline
\end{tabular}

\section{Statistical Analysis}

Given that hundreds of exposures were used in this study, batch MR analyses were implemented using our own script. Here, we upload the core codes of batch two-sample MR analysis in GitHub (https://github.com/zxwang2019/Two-sample-MR.git). LD analyses were performed by using the PLINK software (version 1.9) (26). Two-sample MR analyses, including sensitivity analyses, were all performed by using the TwoSampleMR package (version 0.4.22) (27) in $\mathrm{R}$ (version 3.6.1).

\section{RESULTS}

\section{Information}

A total of 39,142 SNPs were significantly associated with the 486 metabolites $\left(P<1 \times 10^{-5}\right)$ in Sample 1. After LD analyses, the number of these SNPs collapsed into 9,485, and the SNPs were relatively independent from each other. Among the 9,485 SNPs, 335 were associated with at least two metabolites, and no SNP was associated with HF or its risk factors (see Methods). We excluded confounding SNPs and compared them with the SNPs in Sample 2 (Figure 1). Finally, 8,656 (94.6\%) SNPs were selected for subsequent analyses. Five metabolites with IV number of less than three or more than 100 were removed in the subsequent MR analyses for stable and reliable statistical results.

\section{MR Analysis Results}

In this study, IVW model was used as the primary method in estimating the causal relationships between the blood metabolites and HF risk. Theoretically, the multiple-testing adjusted threshold $\left(P<1.03 \times 10^{-4}\right)$ was used in assessing significance, and no metabolite exceeded the strict threshold in this study (Supplementary Table 2). A total of 22 metabolites comprising 11 known metabolites and 11 unknown metabolites showed nominally significant relation $\left(P>1.03 \times 10^{-4}\right.$ but $P<0.05$, IVW method) to HF (Table 1). In the results of the pathway analysis of the 11 known metabolites, we found that the "Valine, leucine, and isoleucine biosynthesis" metabolic pathway that involves L-Isoleucine was significant $(p=0.026)$. $\mathrm{L}$-isoleucine is an essential amino acid and must be supplemented in the diet. A study (28) had shown that the concentration of essential amino acids (including L-isoleucine) in the serum of chronic heart failure patients was significantly lower than that of the control group, suggesting that L-isoleucine may be associated with HF progression. As for the enrichment analysis, however, we did not identify significant $(p<0.05)$ metabolite sets (Supplementary Tables 3-5 and Supplementary Figures 1-3).

What we found that 4-vinylphenol sulfate (OR [95\%CI], $1.22[1.07-1.38]$ ), linolenate (alpha or gamma; [18:3n3 or 6]) (OR [95\%CI], 1.71 [1.16-2.52]), oleoylcarnitine (OR [95\%CI], 1.60 [1.13-2.28]), isoleucine (OR [95\%CI], 2.08 [1.18-3.67]), epiandrosterone sulfate (OR [95\%CI], 1.22 [1.01-1.49]), and deoxycholate (OR [95\%CI], 1.11 [1.00-1.24]) presented potentially increased HF risk, and cysteine-glutathione disulfide (OR [95\%CI], 0.82 [0.710.96]), glycodeoxycholate (OR [95\%CI], 0.90 [0.82-0.98]), 1-arachidonoylglycerophosphoethanolamine (OR [95\%CI], 0.72 [0.54-0.97]), phosphate (OR [95\%CI], 0.43 [0.20-0.94]), and 5dodecenoate $(12: \ln 7)$ (OR [95\%CI], 0.83 [0.70-1.00]) presented potentially decreased HF risk. Furthermore, we added four other models (see Methods) to estimate the causal effects between the 11 potentially HF-related metabolites and HF risk (Table 2). Two metabolites were significant in at least three MR models and showed consistent causal effects in all models (Table 2 and Figure 2), namely, 1-arachidonoylglycerophosphoethanolamine $\left(P_{\text {IVW }}=3.05 \times 10^{-2}, P_{\text {MR Egger }}=2.48 \times 10^{-2}, P_{\text {Weighted median }}\right.$ $=1.4 \times 10^{-2}, P$ Simple mode $=1.2 \times 10^{-1}, P$ Weighted mode $=$ $\left.6.72 \times 10^{-2}\right)$ and 4 -vinylphenol sulfate $\left(P_{\text {IVW }}=2.16 \times 10^{-3}\right.$, $P_{\text {MR Egger }}=1.69 \times 10^{-1}, P_{\text {Weighted median }}=3.34 \times 10^{-3}, P$ Simple mode $=8.7 \times 10^{-2}, P$ Weighted mode $=2.37 \times 10^{-2}$ ). For the 4 -vinylphenol sulfate, the overall results were similar for the five methods/models. The point estimate from MR-Egger regression was similar to this from IVW, and the interval estimates were relatively wide (Figure $\mathbf{2 A}$ ). We noted that there may be an outlier here, while the funnel plot (Supplementary Figure 4) showed that the number of points was almost symmetrically distributed when using individual SNPs as IVs (6 vs. 4). But the corresponding causal effect values were less evenly distributed in the IVW and MR-Egger regression models, suggesting that the results obtained using these 10 SNPs as IVs may still be subject to potential bias.

\section{Evaluation of the Reliability and Stability of the Results}

We performed heterogeneity and horizontal pleiotropy tests on the 11 known metabolites $(P<0.05$, IVW method) to evaluate the reliability and stability of the results. The $P$-values of the test results (including MR-Egger and MR-PRESSO methods) were more than 0.05 and the intercept of MR-Egger regression 
A

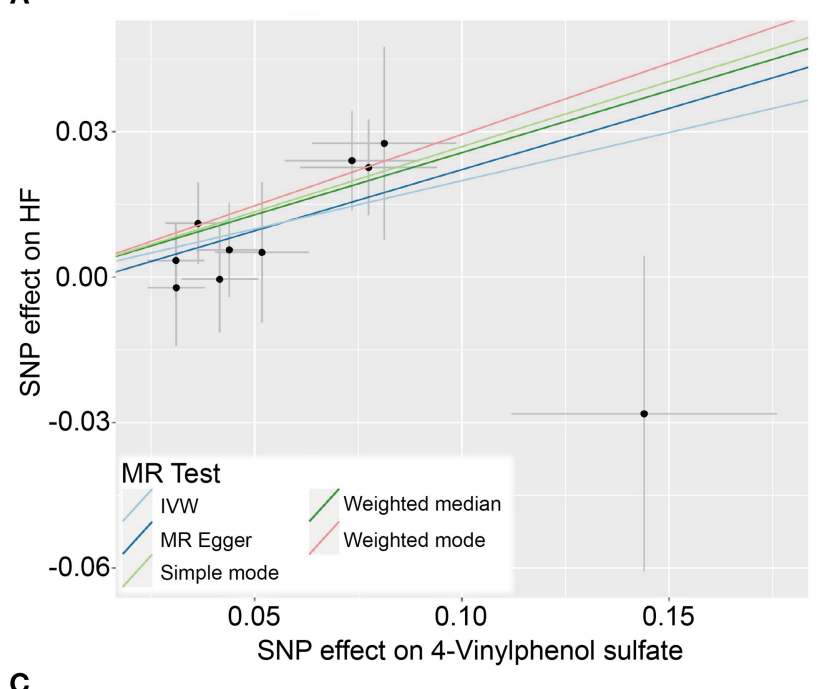

C

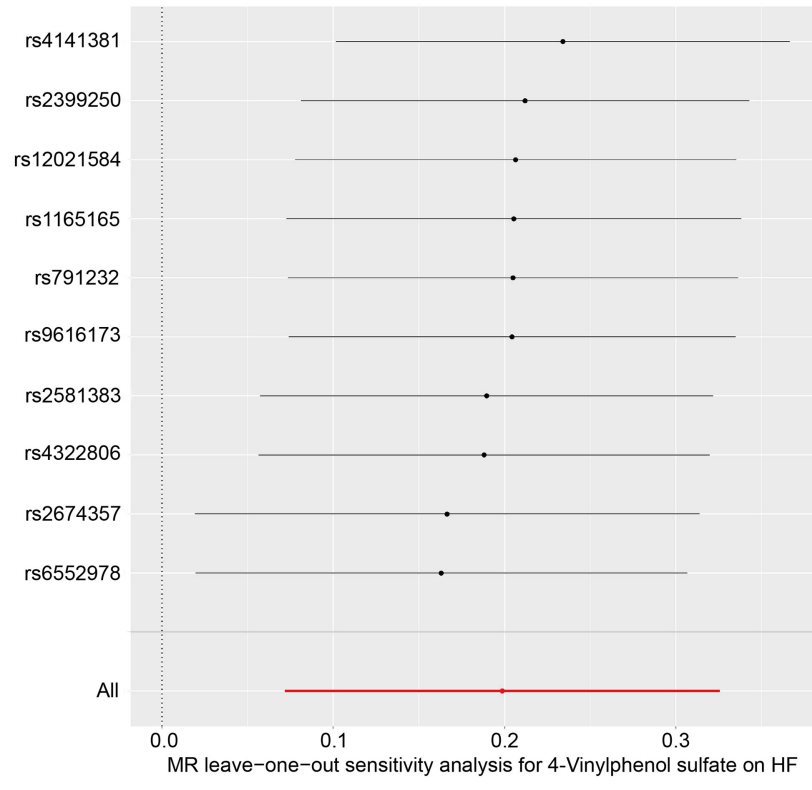

E

\begin{tabular}{ccccc}
\hline Metabolite & Method & nSNP & P & OR (95\%Cl) \\
\hline & MR Egger & 13 & $5.99 E-01$ & $0.52(0.05-5.54)$ \\
& Weighted median & 13 & $9.21 \mathrm{E}-01$ & $1.02(0.64-1.64)$ \\
1-Arachidonoylglycerophosphoethanolamine & IVW & 13 & $8.58 \mathrm{E}-01$ & $0.97(0.67-1.40)$ \\
& Simple mode & 13 & $4.85 \mathrm{E}-01$ & $1.34(0.60-2.97)$ \\
& Weighted mode & 13 & $4.92 \mathrm{E}-01$ & $1.31(0.62-2.80)$ \\
\hline
\end{tabular}

B

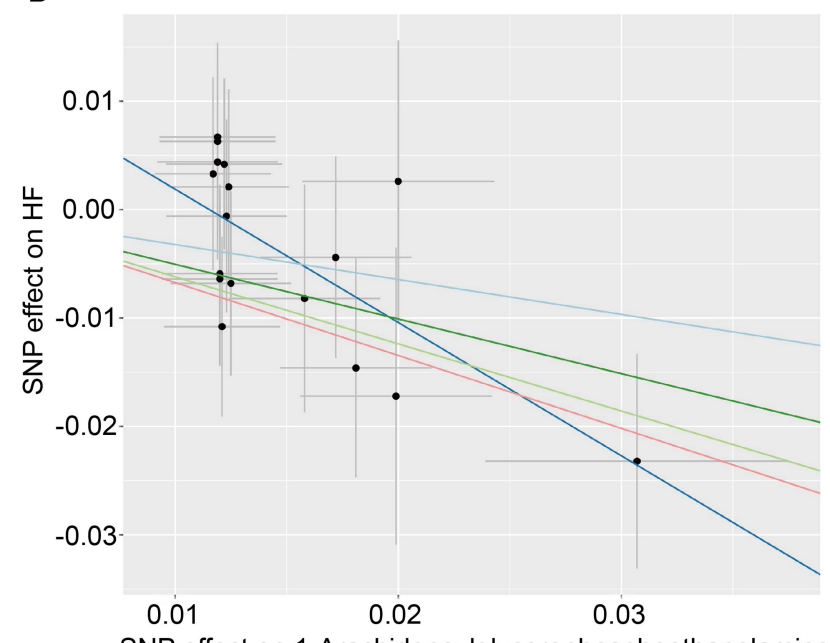

D

SNP effect on 1-Arachidonoylglycerophosphoethanolamine

rs 1984049

rs17031728

rs 11081670

rs39741

rs9624896

rs 4257232

rs35544

rs7599943

rs 10044369

rs 1161762

rs6722402

rs4530945

rs 2504453

rs 2033371

rs1010377

rs7307046

rs17152841

All

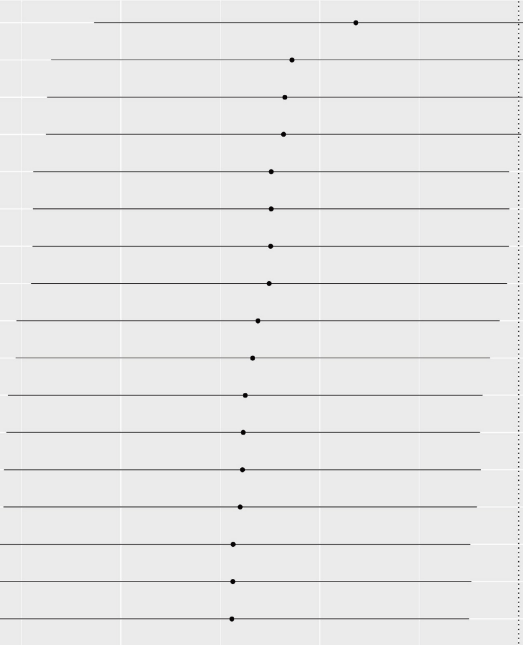

$\begin{array}{lll}-0.50 & -0.25 & 0.00\end{array}$

MR leave-one-out sensitivity analysis for

1-Arachidonoylglycerophosphoethanolamine on HF

FIGURE 2 | Two metabolites showing causal relationships with HF and their subsequent sensitivity analyses. (A,B) Scatter plots of the 5 MR models (light blue line, inverse variance weighted; blue line, MR Egger; light green line, simple model-based estimator; green line, weighted median estimator; red line, weighted model-based estimator) for 4-vinylphenol sulfate and 1-arachidonoylglycerophosphoethanolamine with the risk of HF. (C,D) Forest plots show the results of leave-one-out analyses of the two metabolites. (E) Re-analyses results of five MR models after the removal of sensitive SNP for 1-arachidonoylglycerophosphoethanolamine.

is close to $0(<0.1)$, suggesting evidence of the existence of heterogeneity and horizontal pleiotropy in these metabolites is non-existent (Table 2 and Supplementary Table 6). As for the two relatively robust metabolites (significant in at least three MR models, 1-arachidonoylglycerophosphoethanolamine, and 4-vinylphenol sulfate), we performed sensitivity analyses 


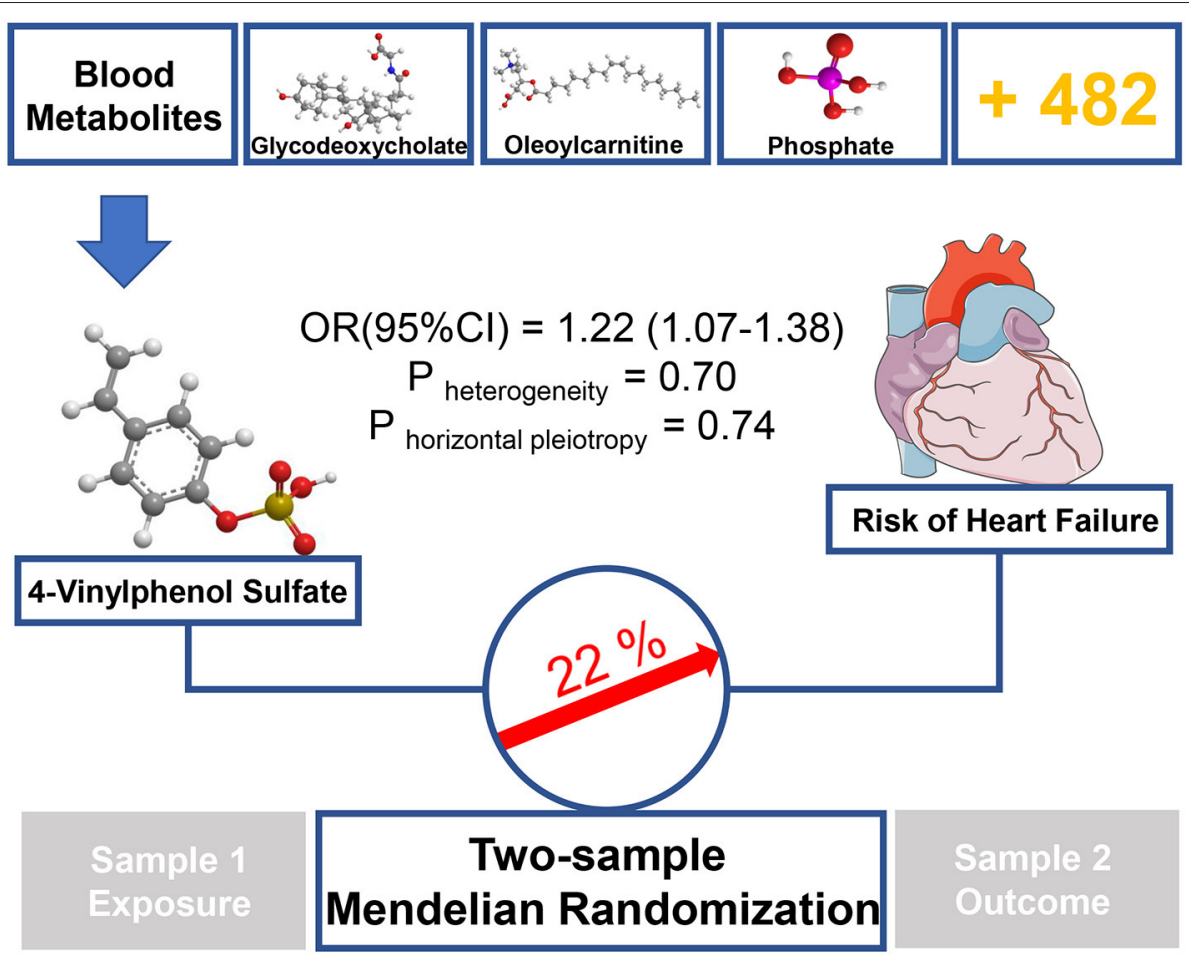

FIGURE 3 | Graphical summary. Among 486 human blood metabolites, this study found that a 1-SD increase in the xenobiotics 4-vinylphenol sulfate was associated with $\sim 22 \%$ higher risk of HF by using two-sample MR approach.

by using a leave-one-out approach to test the stability. All IVs (SNPs) of 4-vinylphenol sulfate showed no sensitivity to the results, suggesting a strong link between exposure and outcome, whereas the four IVs (rs1984049, rs17031728, rs11081670, and rs39741) of 1-arachidonoylglycerophosphoethanolamine may have significantly affected the result (Figures 2C,D). After removing the four sensitive SNPs, we performed MR analyses again using the five models, and we found that the results were no longer significant (Figure 2E).

\section{DISCUSSION}

In this study, we performed unbiased two-sample MR analysis to perform causal evaluation on 486 blood metabolites and $\mathrm{HF}$ risk. We collected the largest mGWAS and large $\mathrm{HF}$ GWAS summary data from public databases. We used genetic variants as IVs and discovered 11 known metabolites, which were considered potential risk predictors of HF after primary IVW analysis. Moreover, to further ensure the reliability and stability of the results, another four MR models and sensitivity analysis were performed. The result consistently supported that the xenobiotic 4-vinylphenol sulfate is related to increased HF risk (see Figure 3).

As a sulfate conjugate, 4-vinylphenol sulfate is one of the main metabolisms of 4-vinylphenol in vivo (29). Naturally found in crops, such as peanut and wild rice (30), 4-vinylphenol is an essential ingredient widely used in meat and seafood flavor formulations (PubChem CID: 62453). Our findings showed that 4-vinylphenol sulfate could increase the incidence of $\mathrm{HF}$ by $22 \%$ (IVW method), suggesting that long-term or excessive diets containing such compound or 4-vinylphenol, especially in the additives, may increase the likelihood of HF. Previous studies have shown that the level of 4-vinylphenol sulfate in the blood is closely related to smoking (31), which is a key risk factor for myocardial systolic dysfunction and hospitalization due to mental failure (32). Petersen et al. (33) showed a significant correlation between 4 -vinylphenol sulfate and methylation at a certain site of $R A R A$, which is a transcription factor that regulates differentiation and apoptosis (34), and the evidence may be linked to the pathogenesis of HF.

Another metabolite is worth mentioning, namely, 1arachidonoylglycerophosphoethanolamine, which can be referred to as LysoPE [20:4 (5Z,8Z,11Z,14Z)/0:0] or LPE (20:4/0:0). After analysis by the primary IVW method, it is found that the metabolite was related to decreased risk of $\mathrm{HF}$ and showed a significant $(P<0.05)$ causation with $\mathrm{HF}$ in another two MR models. However, it did not pass the final leave-one-out analysis. LysoPE [20:4 (5Z,8Z,11Z,14Z)/0:0] is an endogenous compound and a kind of lysolipid. Gao et al. (35) found that LysoPE 20:4 is significantly related to Qi deficiency syndrome in the treatment of congestive HF with traditional Chinese medicine, suggesting that it may be one of the specific metabolic biomarkers of congestive HF treated using traditional Chinese medicine granules. In addition, $\mathrm{HF}$ is associated 
with significant disturbances in phospholipid metabolism. A statistically significant decline in LysoPE level was found in patients with chronic HF with reduced ejection fraction (36). Supplementation with LysoPE in mammalian cells can reverse mitochondrial impairments (37). Our findings suggested that LysoPE 20:4 has a potential positive influence on HF risk, providing an interesting and valuable evidence for future studies.

\section{Innovations and Limitations}

Our study has some innovations. First, from the perspective of molecular mechanism, regarding blood metabolites as exposure factors in exploring the causal relationships between metabolites and HF risk has a solid theoretical basis and important clinical research value. Second, the study used strict quality control conditions and reasonable analysis methods, including a variety of models, to evaluate the causal effects. Thus, the results of this study are reliable and stable. Third, unlike in previous MR analyses of single exposure factors, analysis of a large number of blood metabolites may require huge workloads and present analytical challenges. The analysis strategy we presented might provide a reference for similar studies. Our study may have some limitations. To begin with, all the mGWAS and HF GWAS data were obtained from the European population, and thus comprehensive studies involving different ethnic groups are needed. Furthermore, half of the risk predictors of HF obtained by preliminary analysis (IVW only) are unknown metabolites, and their functional structures are unclear. Thus, the findings in the study are limited. Finally, although we revealed that 4-vinylphenol sulfate is nominal causal related to heart failure by using an unbiased two-sample MR approach, while this relationship was theoretical and we failed to confirm it mechanistically. Hence, further work is still needed to uncover the role of 4-vinylphenol sulfate in the pathogenesis of HF, therefore confirming this causal relationship.

\section{CONCLUSIONS}

In conclusion, we used a two-sample MR approach to explore the causal relationships between 486 blood metabolites and HF

\section{REFERENCES}

1. Groenewegen A, Rutten FH, Mosterd A, Hoes AW. Epidemiology of heart failure. Eur J Heart Fail. (2020) 22:1342-56. doi: 10.1002/ejhf.1858

2. Murphy SP, Ibrahim NE, Januzzi JL Jr. Heart failure with reduced ejection fraction: a review. JAMA. (2020) 324:488-504. doi: 10.1001/jama.2020. 10262

3. Lindgren MP, PirouziFard M, Smith JG, Sundquist J, Sundquist K, Zöller B. A Swedish nationwide adoption study of the heritability of heart failure. JAMA Cardiol. (2018) 3:703-10. doi: 10.1001/jamacardio.2018.1919

4. Shah S, Henry A, Roselli C, Lin H, Sveinbjörnsson G, Fatemifar G, et al. Genome-wide association and Mendelian randomisation analysis provide insights into the pathogenesis of heart failure. Nat Commun. (2020) 11:163. doi: 10.1038/s41467-019-13690-5

5. Cheng ML, Wang CH, Shiao MS, Liu MH, Huang YY, Huang CY, et al. Metabolic disturbances identified in plasma are associated with outcomes in patients with heart failure: diagnostic and prognostic value of metabolomics. J Am Coll Cardiol. (2015) 65:1509-20. doi: 10.1016/j.jacc.2015.02.018 among more than 0.9 million Europeans. We found that 1-SD increase in the xenobiotic 4-vinylphenol sulfate could nominally increase the risk of $\mathrm{HF}$ by $22 \%$. Our findings strengthen our knowledge of the relationships between blood metabolites and HF, which potentially facilitate the establishment of personalized explanation or markers for biological differences in disease status.

\section{DATA AVAILABILITY STATEMENT}

Publicly available datasets were analyzed in this study. This data can be found here: the Metabolomics GWAS Server: http:// metabolomics.helmholtz-muenchen.de/gwas/; the CVDKP Datasets: http://www.kp4cd.org/datasets/mi.

\section{AUTHOR CONTRIBUTIONS}

ZW designed the study, performed data analysis, and drafted the manuscript. SC performed data analysis, drafted, and revised the manuscript. QZ revised the manuscript. YW, GX, and GG collected the data. WL and JC provided the resources. SZ designed the study, leaded the study, and revised the manuscript.

\section{FUNDING}

This work was supported by the grants from the National Nature Science Foundation of China (Nos. 81872934 and 81673514), the Key research and development program of Guangdong Province, China (No. 2019B020229003), Science and Technology Development Projects of Guangdong Province, China (No. 2017B0303314041), and Guangdong Provincial People's Hospital Clinical Research Fund (Y012018085).

\section{SUPPLEMENTARY MATERIAL}

The Supplementary Material for this article can be found online at: https://www.frontiersin.org/articles/10.3389/fcvm. 2021.695480/full\#supplementary-material
6. Lanfear DE, Gibbs JJ, Li J, She R, Petucci C, Culver JA, et al. Targeted Metabolomic profiling of plasma and survival in heart failure patients. JACC Heart Fail. (2017) 5:823-32. doi: 10.1016/j.jchf.2017.07.009

7. Hunter WG, Kelly JP, McGarrah RW, 3rd, Khouri MG, Craig D, Haynes $\mathrm{C}$, et al. Metabolomic profiling identifies novel circulating biomarkers of mitochondrial dysfunction differentially elevated in heart failure with preserved versus reduced ejection fraction: evidence for shared metabolic impairments in clinical heart failure. J Am Heart Assoc. (2016) 5:e003190. doi: 10.1161/JAHA.115.003190

8. Yang J, Yan B, Zhao B, Fan Y, He X, Yang L, et al. Assessing the causal effects of human serum metabolites on 5 major psychiatric disorders. Schizophr Bull. (2020) 46:804-13. doi: 10.1093/schbul/sbz138

9. Geelhoed B, Börschel CS, Niiranen T, Palosaari T, Havulinna AS, Fouodo CJK, et al. Assessment of causality of natriuretic peptides and atrial fibrillation and heart failure: a Mendelian randomization study in the FINRISK cohort. Europace. (2020) 22:1463-9. doi: 10.1093/europace/euaa158

10. Hansen PR, Nelveg-Kristensen KE, Rasmussen HB, Torp-Pedersen C, Køber L, Nielsen CH, et al. Prognostic role of genetic polymorphisms of 
the interleukin-6 signaling pathway in patients with severe heart failure. Pharmacogenomics J. (2019) 19:428-37. doi: 10.1038/s41397-019-0068-2

11. den Hoed M, Eijgelsheim M, Esko T, Brundel BJ, Peal DS, Evans DM, et al. Identification of heart rate-associated loci and their effects on cardiac conduction and rhythm disorders. Nat Genet. (2013) 45:621-31. doi: 10.1038/ng.2610

12. Kamada T, Bracker CE, Bartnicki-Garcia S. Chitosomes and chitin synthetase in the asexual life cycle of Mucor rouxii: spores, mycelium and yeast cells. $J$ Gen Microbiol. (1991) 137:1241-52. doi: 10.1099/00221287-137-6-1241

13. Shin SY, Fauman EB, Petersen AK, Krumsiek J, Santos R, Huang J, et al. An atlas of genetic influences on human blood metabolites. Nat Genet. (2014) 46:543-50. doi: 10.1038/ng.2982

14. Abecasis GR, Auton A, Brooks LD, DePristo MA, Durbin RM, Handsaker $\mathrm{RE}$, et al. An integrated map of genetic variation from 1,092 human genomes. Nature. (2012) 491:56-65. doi: 10.1038/nature11632

15. Hwang LD, Lawlor DA, Freathy RM, Evans DM, Warrington NM. Using a two-sample Mendelian randomization design to investigate a possible causal effect of maternal lipid concentrations on offspring birth weight. Int J Epidemiol. (2019) 48:1457-67. doi: 10.1093/ije/dyz160

16. Burgess S, Butterworth A, Thompson SG. Mendelian randomization analysis with multiple genetic variants using summarized data. Genet Epidemiol. (2013) 37:658-65. doi: 10.1002/gepi.21758

17. Bowden J, Davey Smith G, Burgess S. Mendelian randomization with invalid instruments: effect estimation and bias detection through Egger regression. Int J Epidemiol. (2015) 44:512-25. doi: 10.1093/ije/dyv080

18. Bowden J, Davey Smith G, Haycock PC, Burgess S. Consistent estimation in Mendelian randomization with some invalid instruments using a weighted median estimator. Genet Epidemiol. (2016) 40:304-14. doi: 10.1002/gepi.21965

19. Hartwig FP, Davey Smith G, Bowden J. Robust inference in summary data Mendelian randomization via the zero modal pleiotropy assumption. Int $J$ Epidemiol. (2017) 46:1985-98. doi: 10.1093/ije/dyx102

20. Bowden J, Del Greco MF, Minelli C, Davey Smith G, Sheehan NA, Thompson JR. Assessing the suitability of summary data for two-sample Mendelian randomization analyses using MR-Egger regression: the role of the I2 statistic. Int J Epidemiol. (2016) 45:1961-74. doi: 10.1093/ije/dyw220

21. Verbanck M, Chen CY, Neale B, Do R. Detection of widespread horizontal pleiotropy in causal relationships inferred from Mendelian randomization between complex traits and diseases. Nat Genet. (2018) 50:693-8. doi: 10.1038/s41588-018-0099-7

22. Pang Z, Chong J, Zhou G, de Lima Morais DA, Chang L, Barrette M, et al. MetaboAnalyst 5.0: narrowing the gap between raw spectra and functional insights. Nucleic Acids Res. (2021) 49:W388-96. doi: 10.1093/nar/gkab382

23. Wishart DS, Feunang YD, Marcu A, Guo AC, Liang K, Vázquez-Fresno R, et al. HMDB 4.0: the human metabolome database for 2018. Nucleic Acids Res. (2018) 46:D608-17. doi: 10.1093/nar/gkx1089

24. Frolkis A, Knox C, Lim E, Jewison T, Law V, Hau DD, et al. SMPDB: the small molecule pathway database. Nucleic Acids Res. (2010) 38:D480-7. doi: 10.1093/nar/gkp1002

25. Kanehisa M, Goto S. KEGG: Kyoto encyclopedia of genes and genomes. Nucleic Acids Res. (2000) 28:27-30. doi: 10.1093/nar/ 28.1.27

26. Chang CC, Chow CC, Tellier LC, Vattikuti S, Purcell SM, Lee JJ. Secondgeneration PLINK: rising to the challenge of larger and richer datasets. GigaScience. (2015) 4:7. doi: 10.1186/s13742-015-0047-8

27. Hemani G, Zheng J, Elsworth B, Wade KH, Haberland V, Baird D, et al. The MR-Base platform supports systematic causal inference across the human phenome. Elife. (2018) 7:e34408. doi: 10.7554/eLife.34408.012
28. Tsuji S, Koyama S, Taniguchi R, Fujiwara T, Fujiwara H, Sato Y. Nutritional status of outpatients with chronic stable heart failure based on serum amino acid concentration. J Cardiol. (2018) 72:458-65. doi: 10.1016/j.jjcc.2018.05.007

29. Manini P, De Palma G, Mozzoni P, Andreoli R, Poli D, Bergamaschi E, et al. GSTM1 polymorphism and styrene metabolism: insights from an acute accidental exposure. Toxicol Lett. (2002) 134:201-8. doi: 10.1016/S0378-4274(02)00190-X

30. Leung HW, Ko CH, Yue GG, Herr I, Lau CB. The natural agent 4 vinylphenol targets metastasis and stemness features in breast cancer stem-like cells. Cancer Chemother Pharmacol. (2018) 82:185-97. doi: 10.1007/s00280-018-3601-0

31. Manini P, De Palma G, Andreoli R, Goldoni M, Poli D, Lasagni G, et al. [Urinary excretion of 4-vinyl phenol after experimental and occupational exposure to styrene]. Giornale italiano di medicina del lavoro ed ergonomia. (2003) 25(Suppl. 3):61-2.

32. Kamimura D, Cain LR, Mentz RJ, White WB, Blaha MJ, DeFilippis AP, et al. Cigarette smoking and incident heart failure: insights from the Jackson Heart study. Circulation. (2018) 137:2572-82. doi: 10.1161/CIRCULATIONAHA.117.031912

33. Petersen AK, Zeilinger S, Kastenmüller G, Römisch-Margl W, Brugger M, Peters A, et al. Epigenetics meets metabolomics: an epigenome-wide association study with blood serum metabolic traits. Hum Mol Genet. (2014) 23:534-45. doi: $10.1093 / \mathrm{hmg} / \mathrm{ddt} 430$

34. Hu XX, Zhong L, Zhang X, Gao YM, Liu BZ. NLS-RAR $\alpha$ promotes proliferation and inhibits differentiation in HL-60 cells. Int J Med Sci. (2014) 11:247-54. doi: 10.7150/ijms.6518

35. Gao K, Zhao H, Gao J, Wen B, Jia C, Wang Z, et al. Mechanism of Chinese medicine herbs effects on chronic heart failure based on metabolic profiling. Front Pharmacol. (2017) 8:864. doi: 10.3389/fphar.2017.00864

36. Marcinkiewicz-Siemion M, Ciborowski M, Ptaszynska-Kopczynska K, Szpakowicz A, Lisowska A, Jasiewicz M, et al. LC-MS-based serum fingerprinting reveals significant dysregulation of phospholipids in chronic heart failure. J Pharmaceut Biomed Anal. (2018) 154:354-63. doi: 10.1016/j.jpba.2018.03.027

37. Tasseva G, Bai HD, Davidescu M, Haromy A, Michelakis E, Vance JE. Phosphatidylethanolamine deficiency in Mammalian mitochondria impairs oxidative phosphorylation and alters mitochondrial morphology. J Biol Chem. (2013) 288:4158-73. doi: 10.1074/jbc.M112.434183

Conflict of Interest: The authors declare that the research was conducted in the absence of any commercial or financial relationships that could be construed as a potential conflict of interest.

Publisher's Note: All claims expressed in this article are solely those of the authors and do not necessarily represent those of their affiliated organizations, or those of the publisher, the editors and the reviewers. Any product that may be evaluated in this article, or claim that may be made by its manufacturer, is not guaranteed or endorsed by the publisher.

Copyright $\odot 2021$ Wang, Chen, Zhu, Wu, Xu, Guo, Lai, Chen and Zhong. This is an open-access article distributed under the terms of the Creative Commons Attribution License (CC BY). The use, distribution or reproduction in other forums is permitted, provided the original author(s) and the copyright owner(s) are credited and that the original publication in this journal is cited, in accordance with accepted academic practice. No use, distribution or reproduction is permitted which does not comply with these terms. 